\title{
Computer training program for elimination of instrument distortions
}

\section{Lidia Luizova, Alexei Soloviev}

Lidia A. Luizova, Alexei Soloviev, "Computer training program for elimination of instrument distortions," Proc. SPIE 4588, Seventh International Conference on Education and Training in Optics and Photonics, (28 May 2002); doi: 10.1117/12.468737

SPIE Event: Education and Training in Optics and Photonics 2001, 2001, Singapore, Singapore 


\title{
Computer training program for elimination of instrument distortions
}

\author{
Lidia A. Luizova.*, Alexei V. Soloviev** \\ Petrozavodsk State University
}

\begin{abstract}
A training computer program for simulation and elimination of instrument distortions is described. The program demonstrates the noise influence on the result of instrument distortion elimination. There is an opportunity to reduce noise effects introducing a priory information about the function to be found by filtration of restored function Fourier transform. But when spread function is wide, the distortion elimination becomes impossible. Some applications of the program to simulating and real experiments for educational and scientific purposes in spectroscopy are presented. The program as well as program description and user guide is accessible for everyone on Web site dfe3300.karelia.ru.
\end{abstract}

Keywords: training, computer program, instrument distortions, noise, Fourier transform, simulating experiment.

\section{INTRODUCTION}

If a student, who is good at the principles of optics, is asked, what is the reason of limited optical instrument resolution, the answers might be " light diffraction" or "uncertainty principle". The same idea can be found in textbooks on optics ${ }^{1}$. But it is correct only for Rayleigh's resolution criterion, which is useful for visual observation of images. Most modern optical instruments have photoelectronic output, images are converted to digital form and processed by computers. High power of modern computers enables to suggest that if the spread function ( the image of a point) is known, the instrument distortions may be eliminated. (Sometimes this process is referred to as "reduction to ideal instrument"). Then what is the real reason that limits instrument resolving power? Perhaps one can refuse from expensive instruments with large aperture and receive high resolution by an appropriate calculating algorithm realized by a powerful computer? But the experimental errors or noises often frustrate such attempts. A "reduction to ideal instrument" is the so called improperly posed problem and the solution in presence of noises is impossible without using a priory information about the function to be found. This problem arises not only in optics (spectroscopy and image processing) but in all cases when some function (for example, voltage as a function of time) should be received and instrument distortion cannot be ignored. Future engineers in optics, metrology, electronics, computer science have to understand the essence of this problem, to know how it may be solved and to see the limited nature of computing methods.

The objective of this work is to develop a computer program for educational and scientific applications that allows:

to simulate and graphically demonstrate the instrument distortions,

to eliminate these distortions from simulated functions as well as from functions, received in real experiments, to demonstrate the influence of noise on the result of exclusion of instrument distortions, to show the opportunity of noise effect decrease by using a priory information and to demonstrate the limitations of this method.

The program has an easy and clear interface and was successfully used in educational laboratories in courses on metrology, spectroscopy and plasma diagnostics.

\section{MATHEMATICAL BASIS}

We consider only the instruments that could be described as invariant linear filters. Almost every design for dynamic measurement corresponds to this model in definite limits of input signals and usually one avoids to work outside of these limits.

*alim@karelia.ru; phone 78142761303 ; fax 781427110 00;http:// dfe3300.karelia.ru; Petrozavodsk State University, 33 Lenin str. Petrozavodsk, Russia, 185640; **avsdov@yahoo.com; phone7 8142708200. 
For invariant linear filters the output function $f_{\text {out }}(x)$ is equal to convolution of the input signal $f_{\text {in }}(x)$ with $g(x)$ - spread function or pulse response of this device (the output signal when the input signal is a single pulse or delta -like signal).

$$
f_{\text {out }}(x)=\int f_{\text {int }}\left(x^{\prime}\right) \cdot g\left(x-x^{\prime}\right) d x^{\prime} .
$$

Of course, in real experiments the input and output coordinates are quite different, for example, wave length at the input and the element number of photodetective matrix at the output of spectrometer. But there is always correspondence between input and output coordinates that have been ascertained at the stage of graduation, so that we can use the same variable $\mathrm{x}$ for input and output coordinates. For simplicity, we consider one-dimension functions, but computation may be easy generalized to $2 \mathrm{D}$ case of image processing.

The well known way of solving the integral equation (1) is using the convolution theorem:

$$
\mathrm{F}\left\{\mathrm{f}_{\text {out }}(\mathrm{x})\right\}=\sqrt{2 \pi} \mathrm{F}\left\{\mathrm{f}_{\text {int }}(\mathrm{x})\right\} \cdot \mathrm{F}\{\mathrm{g}(\mathrm{x})\} \text {, }
$$

where $\mathrm{F}$ is Fourier transform operator. If $\mathrm{g}(\mathrm{x})$ is known, we simply have to divide Fourier transform of $\mathrm{f}_{\text {out }}$ to Fourier transform of $g$ and restore $f_{\text {in }}$ by inverse Fourier transform of the result. (this process may be referred to as "deconvolution").

When with noises this method may fail. In this situation we are dealing with a sum of "true " signal $f_{\text {in }}(x)$ and noise $\mathrm{N}(\mathrm{x})$. As a rule, noise spectrum is much wider than pulse response one. As a result, after division of the output function Fourier transform to Fourier transform of spread function we'll receive:

$$
\mathrm{F}^{\prime}\left\{\mathrm{f}_{\text {int }}(\mathrm{x})\right\}=\frac{\mathrm{F}\left\{\mathrm{f}_{\text {out }}(\mathrm{x})\right\}+\mathrm{F}\{\mathrm{N}(\mathrm{x})\}}{\sqrt{2 \pi} \mathrm{F}\{\mathrm{g}(\mathrm{x})\}}=\mathrm{F}\left\{\mathrm{f}_{\text {int }}(\mathrm{x})\right\}+\frac{\mathrm{F}\{\mathrm{N}(\mathrm{x})\}}{\sqrt{2 \pi} \mathrm{F}\{\mathrm{g}(\mathrm{x})\}} .
$$

Even at a low noise level the second term in (3) becomes much greater than the first in the spectral range where Fourier transform of $\mathrm{g}(\mathrm{x})$ tends to zero, and after inverse Fourier transformation of $\mathrm{F}^{\prime}\left\{\mathrm{f}_{\text {in }}(\mathrm{x})\right\}$ we receive only a fast oscillating function and no information about the input signal. Clearly, the simplest way of noise effect diminution is to limit the operative spectral range. The narrower this range the less the noise amplitude in the reconstructed function, but resulting instrument resolution is worse. That's why fine details in input signal would be lost by limitation of spectral range. The constriction of the operative spectral range is equivalent to using a priory information about smoothing degree of the input function.

This property of the process of reduction to ideal instruments is readily illustrated by our program. During the simulating experiments one can be assured that noise sets limits on information about the investigated signals.

\section{BRIEF DESCRIPTION OF THE PROGRAM}

This program was developed by means Borland $\mathrm{C}++5.5$ free command line tools and requires Microsoft Windows 95/98/2000 running on IBM PC AT-compatible with 80386(+387) processor and higher. It also requires at least 400 Kbytes of free memory (depends on precision). It uses system modules KERNEL32.dll, GDI32.dll, USER32.dll, COMDLG32.dll and COMCTL32.dll only.

This program was designed to simulate output signal when input signal and spread function are given and to restore input signal when output signal and spread function are given. The user can simulate noise and can study its influence on the quality of restored signal. The main program window (Fig.1) contains the menu, the toolbar, the graph field and the status window. The program can use both English and Russian versions. The user switches the languages by means of corresponding command from the menu item "Options". The most common commands are placed as buttons on the toolbar: "Function tuning", "Simulation of instrument distortion", "Elimination of instrument distortion", "Copy to clipboard", "Print", "Open a file" and "Save". The user can determine the exact value of one of functions if he chooses it from the list on the toolbar and clicks on the graph of this function. The function value in this point is placed into the status window (ABS - absolute value, $\mathrm{RE}$ - real part, IM - imaginary part). There is current abscissa $(\mathrm{X})$ and the index of function values array for this abscissa (IDX) in the status window as well. The chosen function can be easily scaled (from 1:5 (20\%) up to 5:1 (500\%)) by means of the trackbar of the toolbar. For each chart in the graph field the abscissa axis is drawn with corresponding color. Zero is always located in the center of the graph filed. Each graph presents absolute values of a function. 
The contents of the graph field can be printed or copied to clipboard. Some functions can be hidden or not given. Those functions aren't drawn in the graph field. The user can determine function state in the dialog "Legend". This dialog also allows to change the colors of function graph. The legend can be showed or hidden by means of command "Legend" from the menu item "Options". Calculation starts when the user selects one of the command from the menu item "Calculations". The program places the information about the current step of calculations and its progress into the status window. Calculations can be terminated if a user presses [Escape]. The user can load input signal, spread function or output signal from file. Fourier-images can't be loaded. They can be calculated only. The user can save any function if it is given (or calculated) and visible. The input and output files are texts. The first file line contains the amount of function values, initial value of argument, argument increment and maximal function value. From the second line of the file complex numbers are placed. There is one number in format "real part - imaginary part" in line only. Except loading from a file, function can be defined as internal one. The dialog "Functions tuning" (Fig.2) allows to configure internal functions, to specify precision and to add noise to a signal. The internal input function is the sum of 3 gaussians. The user should specify the amplitude (A), the abscissa of peak (B) and the width (C) of every gaussian. Spread function can be defined as normalized gaussian (the user specifies the width $(C)$ of gaussian and the program recalculates the amplitude to normalize the spread function).

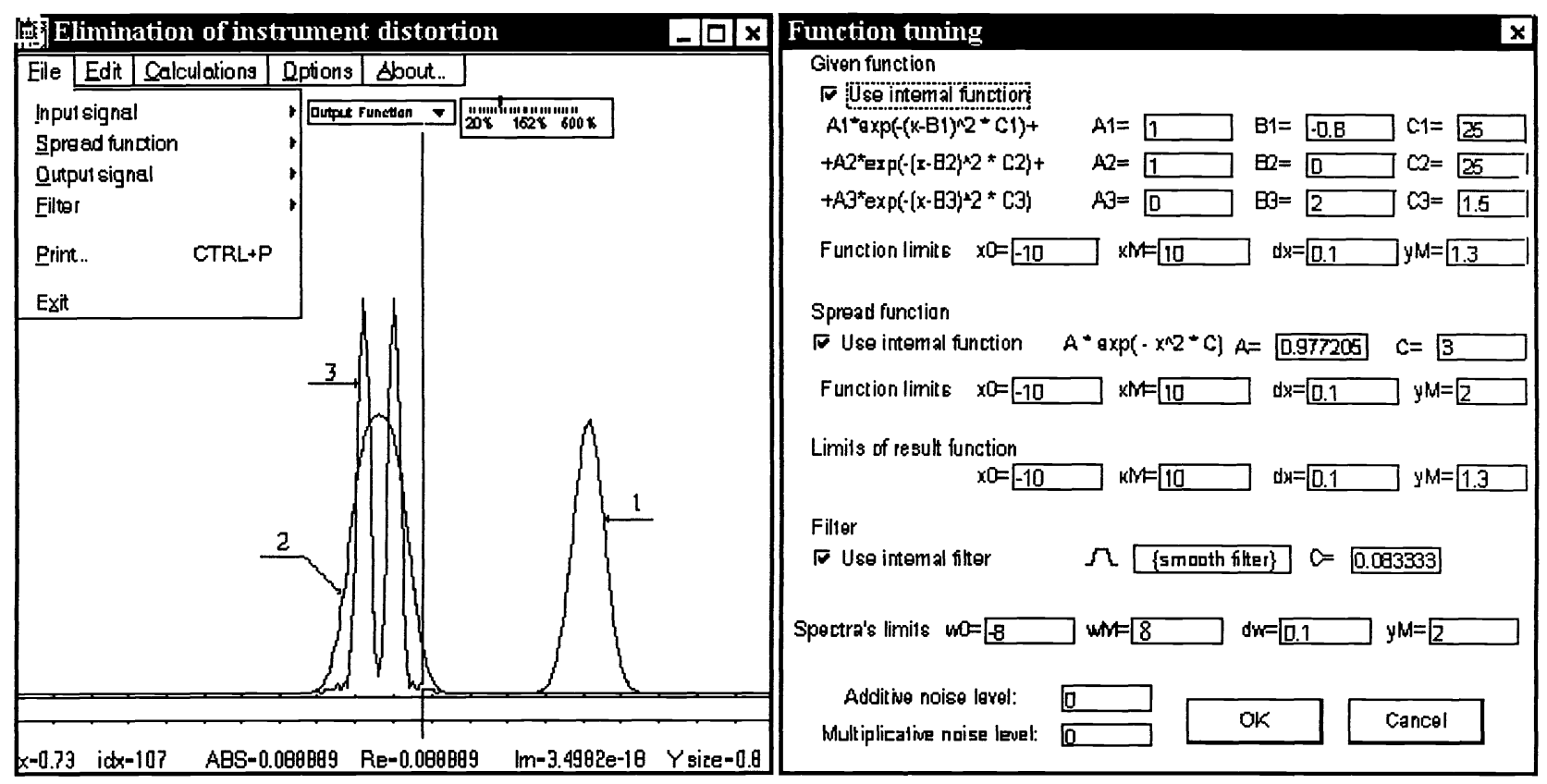

Fig.1: Main program window with submenu "File". 1-spread function, 2-output function, 3- restored function

Fig. 2: The dialog window "Functions tuning"

The program adds two kinds of noises: additive and multiplicative. The user may specify "noise level" $\sigma$ in a corresponding field, then the program changes every calculated value $f$ of the simulated output function to $F=f+y f$, when multiplicative noise is chosen, and to $\mathrm{F}=\mathrm{f}+\mathrm{yY}$ for the additive case, where $\mathrm{y}$ is a normally distributed value with zero mean and variance equal to $\sigma^{2}, Y$ - window size, specified in the field "yM" for result function.

For decreasing the noise influence, one can use filtration of restored function Fourier transform. There are two types of filter selected by the button "filter":

1) binary filter - in this case the restored function Fourier transform, calculated according (3), is multiplied by 1 inside of the limits specified in fields "Spectral limits", it is equal to zero outside of this limits ;

2) smooth filter - at this case Fourier transform is also multiplied by 1 inside of "Spectral limits", but outside them Fourier transform is multiplied by exponential decreasing value with an index specified in the field " $\mathrm{C}$ ". 


\section{LABORATORY TRAINING WITH THE PROGRAM: SIMULATING EXPERIMENTS}

\subsection{Overcoming Rayleigh's resolution criterion}

If students simulate an input function as two clearly separate peaks ( for example, spectral doublet) and spread function as a gaussian with width comparable with peak separation, they'll receive an output function with no gap, so by visual observation two lines could not be resolved (Fig.1). Restoring input function by the program one can receive almost correct input signal. Small oscillations of restored signal will be discussed later. (For clearness in all pictures ordinate scales for functions are different and the spread function is shifted sometimes from its "natural" place in the program window)

\subsection{Effect of noises}

Simulating an input and a spread function and calculating the convolution students can add to the output function a specified form and level of noises, and then try to restore the input signal. Even with low values of noises they may receive senseless results (Fig 3.) They can decrease the noise effect by reducing the operative spectral range. (Fig 4.)

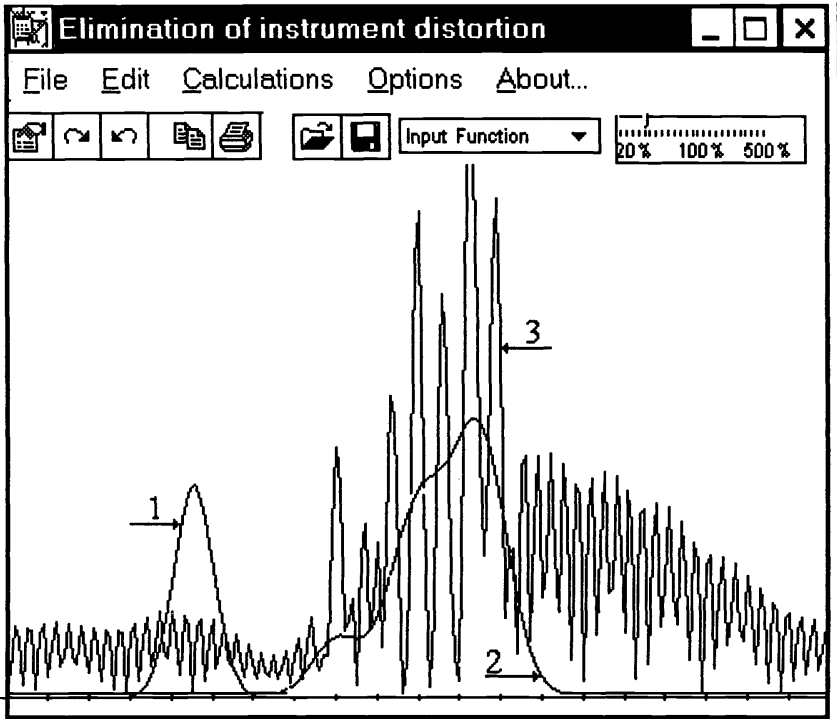

Fig.3: Elimination of instrument distortion with noises. $\sigma=0.0001$, additive noise. 1-spread function, 2-output function, 3- restored function

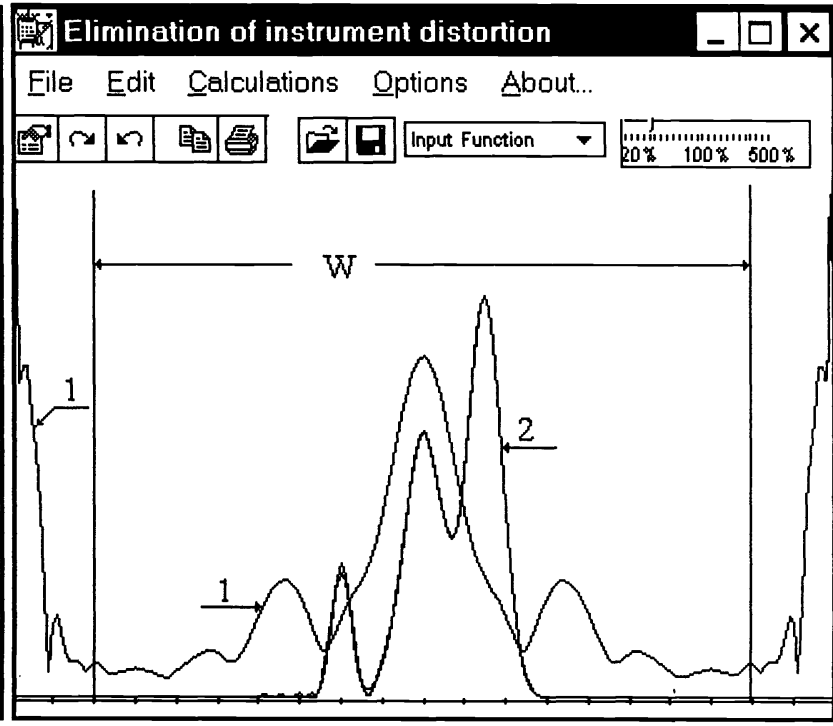

Fig. 4: Noise level, spread function, and output functions are the same as in Fig. 3, but operative spectral range is reduced to $\mathrm{W}$ (0.8 of initial range). 1- restored function spectrum. 2restored and simulated "true" functions are almost identical

Then students have to investigate the effect of spread function width on the result of reduction to ideal instrument. They may see that if the spread function is wide and the noise level is not low the slight decreasing of the operative spectral range (to 0.6 of initial range) does not improve the picture (Fig 5). The oscillations disappear with the narrow operative spectral range ( 0.3 of initial value), but if the spread function is too wide, the restored function is now more similar to the output function than to the input one, that is to say the instrument distortion elimination in this case is impossible. (Fig.6). Even if specified noise level is zero, the limited accuracy of computer calculation has an effect like noises (this is the reason of restored function oscillations in Fig. 1).

So students have to understand that inevitable noise puts the real limit for optical instruments resolution. The better is the instrument and the narrower is its spread function the lower are the demands to noise level.

\subsection{Rearrangement of operation in plasma spectroscopy}

When studying the course on plasma diagnostics students have to master Abel's inversion methods for determination local plasma spectral emission. The method applied to an array of spectral line profiles, received by the instrument of 
limited resolution, demands prior elimination of instrument distortions. Simulating experiment with the described program and the program for simulating arrays of "true" line profiles, excited in high pressure discharge 6 , allows to show that the order of deconvolution and Abel's inversion may be interchanged.

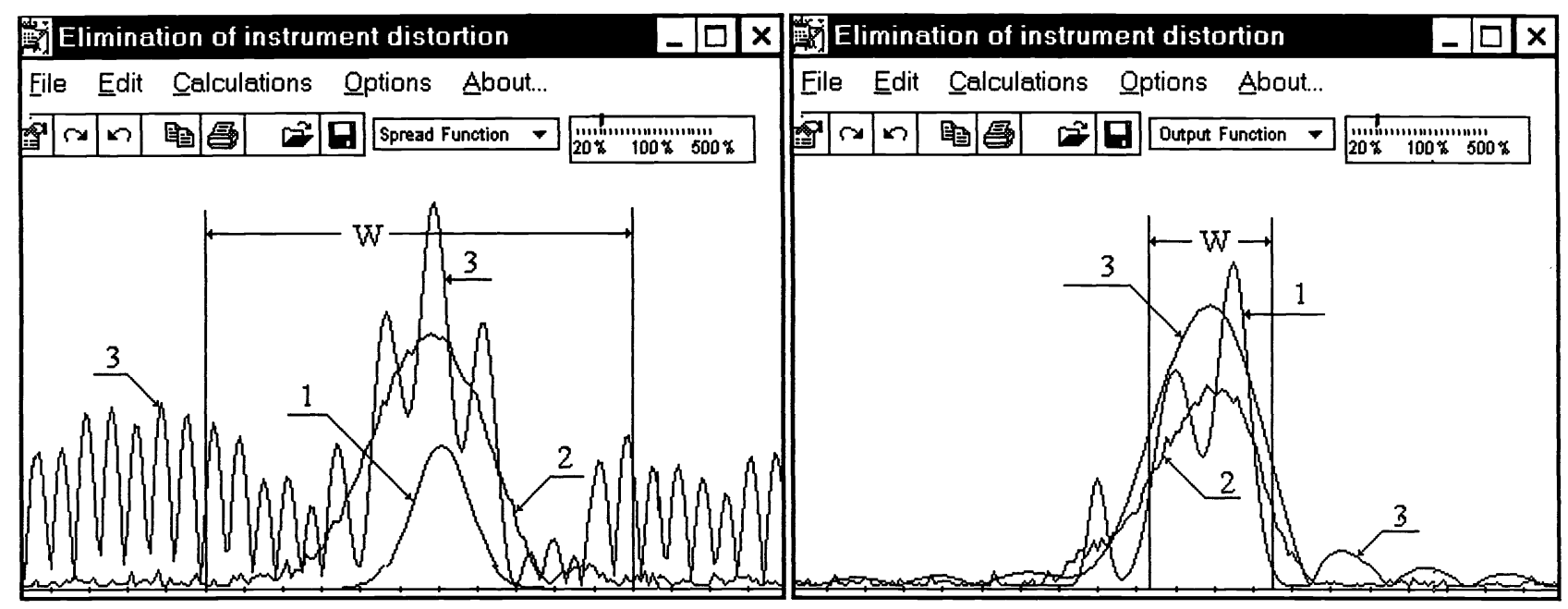

Fig. 5: Elimination of instrument distortion with noises $\sigma=0.01$ and wide spread function (1), 2-output function, 3- restored function
Fig. 6: Decreasing of oscillations by reducing operative spectral range to W. Spread function as in Fig 5. 1- simulated "true" function, 2-output function, 3- restored function.

To investigate one section of cylindrical symmetric plasma, two-dimensional array of visible spectral intensities $b\left(\lambda_{i}, y_{k}\right)$ has to be measured along a series of chords, perpendicular to plasma axis ( $y_{k}-$ the displacement of chord from the plasma centerline, $\lambda_{i}$ - the wavelength of spectral point inside the profile). From the array of visible intensities $b\left(\lambda_{i}, y_{k}\right)$ the array of spectral emissivities $\varepsilon\left(\lambda_{i}, r_{k}\right)$ has to be reconstructed and spectral line profiles $\varepsilon\left(\lambda_{i}, r_{k}\right)$ for distance $r_{k}$ from section center may be used for determination of plasma parameters in this spatial point.

The relation between $b\left(\lambda_{i}, y_{k}\right)$ and $\varepsilon\left(\lambda_{i}, r_{k}\right)$ is given by: ${ }^{2}$

$$
\mathrm{b}(\lambda, \mathrm{y})=2 \int_{\mathrm{y}}^{\mathrm{R}} \frac{\varepsilon(\lambda, \mathrm{r}) \mathrm{rdr}}{\sqrt{\mathrm{r}^{2}-\mathrm{y}^{2}}}=A\{\varepsilon(\lambda, \mathrm{r})\}
$$

(R-radius of plasma edge). The reconstruction of emissivity $\varepsilon(r)$ from the measured intensity $b(x)$ is known as Abel's inversion. ${ }^{2,3}$

$$
\varepsilon_{\lambda}(\mathrm{r})=-\frac{1}{\pi} \int_{\mathrm{r}}^{\mathrm{R}} \frac{\mathrm{db}_{\lambda}(\mathrm{x})}{\mathrm{dx}} \frac{\mathrm{dx}}{\sqrt{\left(\mathrm{x}^{2}-\mathrm{r}^{2}\right)}}=A^{-1}\{\mathrm{~b}(\lambda, \mathrm{y})\} .
$$

Here $A$ denotes the transformation operator $\varepsilon(\lambda, \mathrm{r})$ to $\mathrm{b}(\lambda, \mathrm{y})$ and $A^{-1}$-the inverse one. In real experiments the intensity profile $b(\lambda, y)$ may be distorted by instrument pulse response $g(\lambda)$. Consequently, the measured profile at each y is according to (1):

$$
\mathrm{f}_{\text {out }}(\lambda, \mathrm{y})=\int \mathrm{b}_{\mathrm{y}}\left(\lambda^{\prime}\right) \cdot \mathrm{g}\left(\lambda-\lambda^{\prime}\right) \mathrm{d} \lambda^{\prime}=C\{\mathrm{~b}(\lambda, \mathrm{y})\}
$$

Here $C$ signifies the operator of convolution. Let us denote the inverse operator (deconvolution, elimination of instrument distortion) by $C^{l}$.

The natural order of operation for receiving the required array $\varepsilon\left(\lambda_{i}, r_{k}\right)$ from the recorded one $f_{\text {out }}\left(\lambda_{i}, y_{k}\right)$ is the following:

a. $\mathrm{b}\left(\lambda, \mathrm{y}_{\mathrm{k}}\right)=C^{-l} \cdot\left\{\mathrm{f}_{\text {out }}\left(\lambda, \mathrm{y}_{\mathrm{k}}\right)\right\}$ for all position $\mathrm{y}_{\mathrm{k}}$,

b. $\varepsilon\left(\lambda_{\mathrm{i}}, \mathrm{r}\right)=A^{-1}\left\{\mathrm{~b}\left(\lambda_{\mathrm{i}}, \mathrm{y}\right)\right\}$ for all spectral points inside the profile ( Further this way will called as "individual Abel's transform").

That is: 


$$
\varepsilon(\lambda, \mathrm{r})=A^{-1}\left\{C^{-1}\left\{\mathrm{f}_{\text {out }}(\lambda, \mathrm{y})\right\}\right\}
$$

However, since the operators $A^{-1}$ and $C^{-1}$ act on different variables, their order may be interchanged, and we have to receive the same result by the calculation:

$$
\varepsilon(\lambda, \mathrm{r})=C^{-1}\left\{A^{-1}\left\{\mathrm{f}_{\text {out }}(\lambda, y)\right\}\right\}
$$

The program, described above, several programs of Abel's transform ${ }^{4,5}$ and plasma emission simulating program ${ }^{6}$ allow students to test this theoretic conclusion in practice by simulating experiment.

The line profiles $b\left(\lambda_{i}, y_{k}\right)$ of thallium spectral line $\lambda=655 \mathrm{~nm}$, excited in high pressure mercury discharge with addition of thallium, are simulated for 10 position $y_{k}$ and 100 spectral points $\lambda_{i}$. Then, array $f_{\text {out }}\left(\lambda_{i}, y_{k}\right)$ was simulated with spread function, that is shown in Fig.7. In simulating experiments without noises the results of reconstruction of "true " profiles $\varepsilon\left(\lambda_{\mathrm{i}}, \mathrm{r}_{\mathrm{k}}\right)$ received by application of orders (7) and (8) for operators $A^{-1}$ and $C^{-1}$ coincide to the fullest. But when with noise, the results depend significantly on noise level, operative spectral range, used for deconvolution and algorithms, used for realization $A^{-1}$ operator. Abel's transform is also an improperly posed problem and a method for decreasing of noise effect is proposed in $^{5}$. It is "combined "Abel's transform, where only Abel's transforms for average spatial distribution $\bar{B}_{k}$ and several eigenvectors $U_{p, k}$ of covariance matrix of experimental array $b\left(\lambda_{i}, y_{k}\right)$ may be fulfilled instead of inversions $A^{-1}$ for each spectral component. The array $\mathrm{b}\left(\lambda_{\mathrm{i}}, \mathrm{y}_{\mathrm{k}}\right)$ is presented as:

$$
b\left(\lambda_{i}, y_{k}\right)=\bar{B}_{k}+\sum_{p=1}^{q} L_{p}\left(\lambda_{i}\right) U_{p, k}
$$

Here $L_{p}\left(\lambda_{i}\right)$ are the projections of $i$-th point of m-dimension space, corresponding to particular $\lambda_{i}$. to direction of $U_{p}$. Abel's transform operator is linear, therefore we can use (9) and write:

$$
A^{-1}\left\{\mathrm{~b}\left(\lambda_{\mathrm{i}}, \mathrm{y}_{\mathrm{j}}\right)\right\}=A^{-1}\left\{\overline{\mathrm{B}}_{\mathrm{k}}\right\}+\sum_{\mathrm{p}=1}^{\mathrm{q}} \mathrm{L}_{\mathrm{p}}\left(\lambda_{\mathrm{i}}\right) A^{-1}\left\{\mathrm{U}_{\mathrm{p}}\right\} .
$$

The number $q$ of eigenvectors that is used in (10) depends on variability of profiles $b\left(\lambda_{i}, y_{k}\right)$ and noise level. (the criteria for choice of $q$ are discussed in ${ }^{5}$ ). Looking at $(9,10)$ more carefully one can see that dependence on $\lambda$ is contained only in $\mathrm{L}_{\mathrm{p}}\left(\lambda_{\mathrm{i}}\right)$. Consequently, we can realize $C^{-1} A^{-1}$ operator by the following way:

a. Finding the average spatial distribution $\bar{B}_{k}$ and eigenvectors $U_{p, k}$ of covariance matrix of experimental array $\mathrm{f}_{\text {out }}\left(\lambda_{\mathrm{i}}, \mathrm{y}_{\mathrm{k}}\right)$.

b. Finding $\mathrm{M}_{\mathrm{p}}\left(\lambda_{\mathrm{i}}\right)$ - the projections of $\mathrm{i}$-th point of m-dimension space, corresponding to particular $\lambda_{\mathrm{i}}$, to direction of $\mathrm{U}_{\mathrm{p}, \mathrm{k}}$

c. Eliminating the instrument distortion only from q functions $\mathrm{M}_{\mathrm{p}}\left(\lambda_{\mathrm{i}}\right)$ as $\mathrm{L}_{\mathrm{p}}\left(\lambda_{\mathrm{i}}\right)=C^{-1}\left\{\mathrm{M}_{\mathrm{p}}\left(\lambda_{\mathrm{i}}\right)\right\}$

d. Restoring "true" profiles $\varepsilon\left(\lambda_{i}, r_{j}\right)$ as

$$
\varepsilon\left(\lambda_{\mathrm{i}}, \mathrm{r}_{\mathrm{j}}\right)=A^{-1}\left\{\overline{\mathrm{B}}_{\mathrm{k}}\right\}+\sum_{\mathrm{p}=1}^{\mathrm{q}} \mathrm{L}_{\mathrm{p}}\left(\lambda_{\mathrm{i}}\right) A^{-1}\left\{\mathrm{U}_{\mathrm{p}}\right\}
$$

In Fig.7-9 the results of simulating experiments are represented. Additive normally distributed noises with the standard deviation equal to $1 \%$ of the greatest element of $f_{\text {out }}\left(\lambda_{i}, y_{k}\right)$ array were added to all values $f_{\text {out }}\left(\lambda_{i}, y_{k}\right)$. It was found that only two eigenvectors are significant. In Fig. 7,8 deconvolutions of projections are presented. (absolute values of functions is drawn, really the left "wings" of $\mathrm{M}_{2}$ and $\mathrm{L}_{2}$ are negative). In Fig. 9 the comparison of "individual Abel's transform" and order of operation (7) with reconstruction of $\varepsilon\left(\lambda_{i}, r_{j}\right)$ according to (11) is presented. This experiment shows that in presence of noises one can reduce computing time and improve stability of results by "combined" Abel's transform and deconvolution of the restricted number of function $M_{p}\left(\lambda_{i}\right)$.

\section{APPLICATION TO REAL EXPERIMENTS}

\subsection{Elimination of the Fabry-Perot spread function}

The well known method of determination of atom temperature in low pressure gas discharges is the measurement of Doppler broadening of spectral lines ${ }^{2}$. The line width $\Delta \lambda$, which is connected with atom temperature $\mathrm{T}$ by relationship:

$\Delta \lambda=\frac{\lambda_{0}}{\mathrm{c}} \sqrt{\frac{2 \ln (2) \mathrm{kT}}{\mathrm{m}}}$, where c-light velocity, $\mathrm{k}$-Boltzmann constant, m-atom masse, $\lambda_{0^{-}}$average wave length of the 
profile, is in the order of $0.01 \mathrm{~nm}$, therefore one has to use high resolution spectrometers, such as Fabry-Perot interferometer.

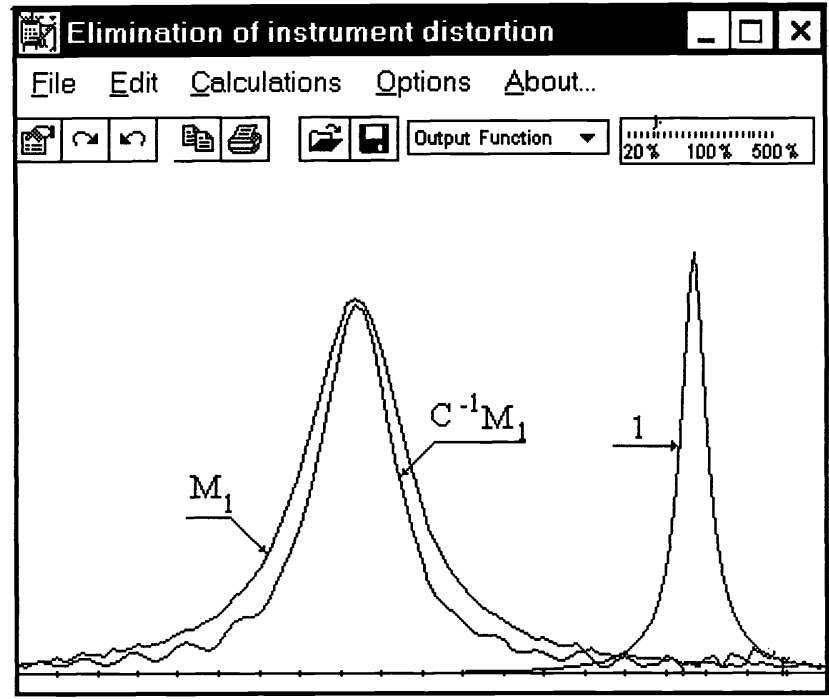

Fig. 7: Projection $M_{1}$ and its deconvolution $C^{-1} M_{1}$, 1 - spread function.

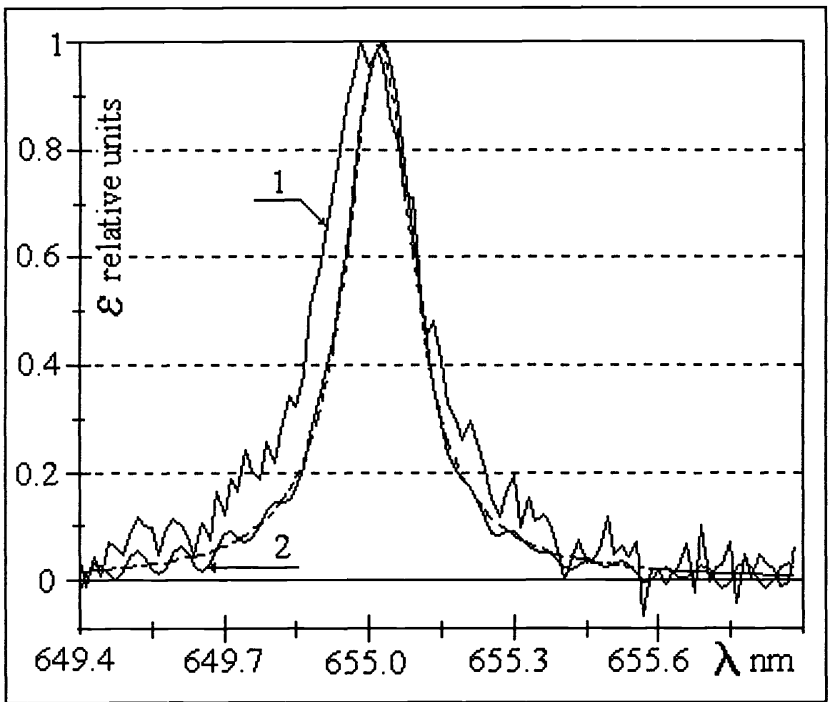

Fig. 9: Thallium line profile for the center point of discharge 1- reconstruction according to order of operation(7) and "individual Abel's transform", 2-combined Abel tranform according to (11). At the most part of profile curve 2 coincide with " true" profile (dotted line).

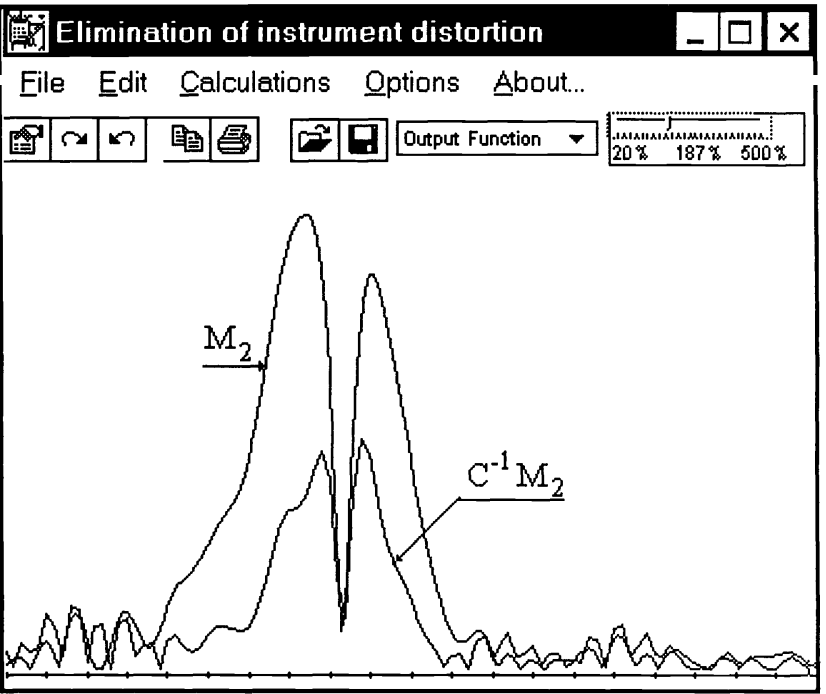

Fig. 8: Projection $\mathrm{M}_{2}$ and its deconvolution $\mathrm{C}^{-1} \mathrm{M}_{2}$,

The pulse response of Fabry-Perot depends on mirrors reflectance $\mathrm{R}$. For high values of $\mathrm{R}$ it may be approximated by:

$$
g(x)=\frac{\gamma}{2 \pi\left(x^{2}+\gamma^{2}\right)}, \gamma=\frac{1-R}{\pi \sqrt{R}},
$$

here $\mathrm{x}$ - relative units, the fraction of the distance between the nearby interference peaks.

$(-0.5 \leq \mathrm{x} \leq 0.5)$

The corresponding values in wave length scale are:

$$
\lambda-\lambda_{0}=\mathrm{x} \cdot \frac{\lambda_{0}^{2}}{2 \mathrm{~d}}, \delta \lambda_{\mathrm{a}}=\gamma \cdot \frac{\lambda_{0}^{2}}{2 \mathrm{~d}},
$$

where $d$ is the distance between the interferometer mirrors. The relationship between the true line width $\Delta \lambda$ and the spread function one $\delta \lambda_{\mathrm{a}}$ depends on $\mathrm{d}$, but the superposition of interference orders limits the increasing of $d$. Therefore almost always the interferometer pulse response has to be taken into account .

In Fig.10 the profiles of the neon spectral line $(\lambda=540 \mathrm{~nm})$ in glow discharge before and after elimination of instrument distortion are presented. Spread function is calculated by students with the known data: $\mathrm{d}=1.2 \mathrm{~cm}, \mathrm{R}=0.9$. In spite of significant noise oscillations, one can see that line width has significantly fallen after elimination of spread function. The temperature calculated from the initial profile by (4) is $(970 \pm 40) \mathrm{K}$, whereas from the restored one we receive only $(640 \pm 30) \mathrm{K}$. 


\subsection{Distortion of self-absorbed line shape}

In one of the laboratory programs on plasma diagnostics students have to compare the simulated by program ${ }^{6}$ and experimental shape of self-absorbed spectral line in emission of inhomogeneous optical dense plasma. If the supposed plasma parameters are correctly chosen and instrument distortion of experimental line shape is negligible satisfactory agreement between real and simulated profiles may be reached ${ }^{6}$. But sometimes an instrument with low resolution (prism spectrometer) is used. In this case expected and received profiles are even qualitatively different, for example, red peak according to theory has to be lower than the blue one, in experiment it is considerably higher. Then the student has to receive the spread function of the instrument by registration some narrow spectral line profile in the same parameters of instrument and imaging system (widths of slits, aperture and so on) and try to eliminate instrument distortion by described program. The example of results for Tl spectral line $(\lambda=535 \mathrm{~nm})$ is presented in Fig. 11 .

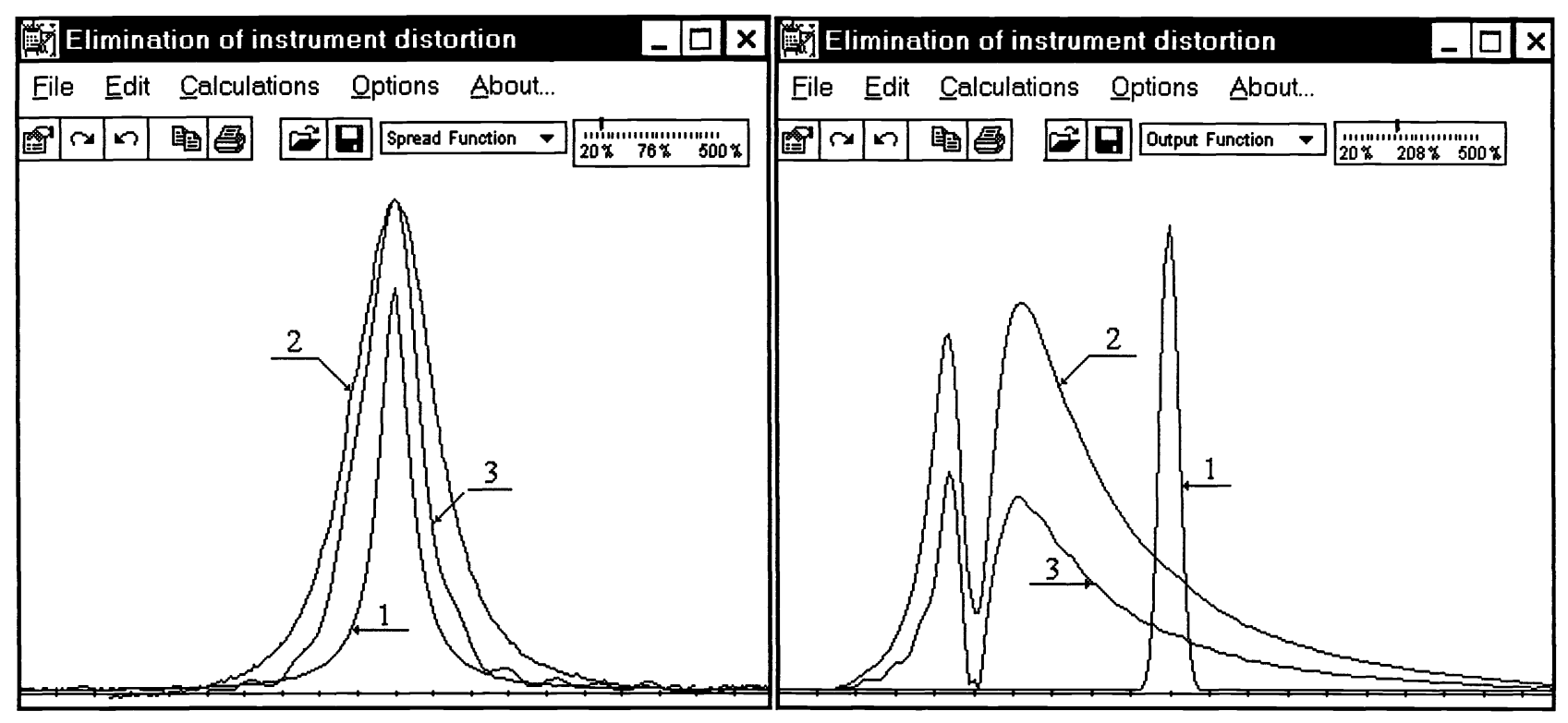

Fig.10: The profile of the neon spectral line before (2) and after (3) elimination of instrument distortion. 1- the interferometer pulse response.
Fig.11: Self-absorbed thallium spectral line before (2) and after (3) elimination of instrument distortion. 1 - the spread function

\section{ACKNOWLEGMENTS}

Authors wish to acknowledge the support of Institute for High Energy Densities of Associated Institute for High Temperature of Russian Academy of Sciences; and to thank Prof. S. D. Wagner and Prof. E. I. Sokolova for useful discussions.

\section{REFERENCES}

1. J.R. Meyer-Arendt, Introduction to Classic and Modern Optics, p.222, Prentice-Hall, New Jersey, 1984.

2. Hans R. Griem, Plasma Spectroscopy, p.151, MCGraw-Hill Book Company, New York, 1964.

3. M.J Buie, J.T.P. Pender, J.P.Holloway, T.Vincent, P.L.G. Ventzek and M. L. Brake, "Abel's inversion applied to experimental spectroscopic data with off axis peaks ", JQSRT, 55, pp. 231-243, 1996.

4. L.A. Luizova, "Well-grounded method of radial transform", Optics and Spectroscopy, 52, pp.690-695, 1982.

5. L.A.Luizova. "Abel's inversion applied to array of spectral line profiles", JQSRT, 66, pp. 277-283, 2000.

6. L.A. Luizova, A. V. Patroev and A. D. Khakhaev, "Simulation of spectral line profiles in emission of closed arc discharge", Jorn. of Applied Spectroscopy, 65, pp.763-770, 1998. 